Draft version September 24, 2014

Preprint typeset using $\mathrm{L}^{A} \mathrm{~T} \mathrm{E}$ X style emulateapj v. 05/12/14

\title{
MODEL INDEPENDENT EVIDENCE FOR DARK ENERGY EVOLUTION FROM BARYON ACOUSTIC OSCILLATIONS
}

\author{
V. Sahni ${ }^{1}$, A. Shafieloo ${ }^{2,3}$, A. A. Starobinsky ${ }^{4,5}$ \\ Draft version September 24, 2014
}

\begin{abstract}
Baryon Acoustic Oscillations (BAO) allow us to determine the expansion history of the Universe, thereby shedding light on the nature of dark energy. Recent observations of BAO's in the SDSS DR9 and DR11 have provided us with statistically independent measurements of $H(z)$ at redshifts of 0.57 and 2.34, respectively. We show that these measurements can be used to test the cosmological constant hypothesis in a model independent manner by means of an improved version of the $\mathrm{Om}$ diagnostic. Our results indicate that the SDSS DR11 measurement of $H(z)=222 \pm 7 \mathrm{~km} / \mathrm{sec} / \mathrm{Mpc}$ at $z=2.34$, when taken in tandem with measurements of $H(z)$ at lower redshifts, imply considerable tension with the standard $\Lambda$ CDM model. Our estimation of the new diagnostic $O m h^{2}$ from SDSS DR9 and DR11 data, namely $O m h^{2} \approx 0.122 \pm 0.01$, which is equivalent to $\Omega_{0 m} h^{2}$ for the spatially flat $\Lambda$ CDM model, is in tension with the value $\Omega_{0 m} h^{2}=0.1426 \pm 0.0025$ determined for $\Lambda$ CDM from Planck+WP. This tension is alleviated in models in which the cosmological constant was dynamically screened (compensated) in the past. Such evolving dark energy models display a pole in the effective equation of state of dark energy at high redshifts, which emerges as a smoking gun test for these theories.
\end{abstract}

Subject headings: expansion history — cosmology: observations - methods: statistical

\section{INTRODUCTION}

There is ample observational evidence to suggest that the expansion of the universe is accelerating, fuelled perhaps by dark energy (DE) which violates the strong energy condition, so that $\rho+3 P<0$. While the cosmological constant with $8 \pi G T_{i k}=\Lambda g_{i k}$ and $P=-\rho \equiv$ $-\Lambda / 8 \pi G$, envisioned by Einstein almost a century ago, fulfills this requirement, the tiny value associated with $\Lambda$ has prompted theorists to look for alternatives in which dark energy evolves with time including modified gravity (Sahni \& Starobinsky 2000; Carroll 2001; Peebles \& Ratra 2003; Padmanabhan 2003; Sahni 2004; Copeland et al. 2006; Sahni \& Starobinsky 2006; Clifton et al. 2012; Shafieloo 2014).

Meanwhile, the very simplicity of the cosmological constant has prompted the search for null-diagnostics which can inform us, on the basis of observations, whether or not DE is the cosmological constant.

One such diagnostic is the Statefinder $r=\dddot{a} / a H^{3}$ (also called the jerk) whose value stays pegged to unity only in ACDM (Sahni et al. 2003; Alam et al. 2003) (also see(Chiba \& Nakamura 1998; Visser 2004)). Thus if observations were to inform us that $r \neq 1$, then this would imply a falsification of the cosmological constant hypothesis.

\footnotetext{
varun@iucaa.ernet.in

arman@apctp.org

alstar@landau.ac.ru

${ }^{1}$ Inter-University Centre for Astronomy and Astrophysics,

Post Bag 4, Ganeshkhind, Pune 411 007, India

2 Asia Pacific Center for Theoretical Physics, Pohang, Gyeongbuk 790-784, Korea

3 Department of Physics, POSTECH, Pohang, Gyeongbuk 790-784, Korea

${ }^{4}$ Landau Institute for Theoretical Physics RAS, Moscow 119334, Russian Federation

${ }^{5}$ Kazan Federal University, Kazan 420008, Republic of Tatarstan, Russian Federation
}

A second null diagnostic, $\operatorname{Om}(z)$, is defined as (Sahni et al. 2008; Zunkel \& Clarkson 2008)

$$
O m(z)=\frac{\tilde{h}^{2}(z)-1}{(1+z)^{3}-1}, \quad \tilde{h}=H(z) / H_{0} .
$$

A remarkable feature of $\mathrm{Om}$ is that its value remains pegged to $\Omega_{0 m}$ in $\Lambda \mathrm{CDM}$. In all other DE models the value of $O m(z)$ evolves with time.

While the Statefinder has proved exceedingly versatile in differentiating between rival DE models, a distinguishing feature of $O m$ is that it depends only upon the expansion rate, $H(z)$, and is therefore easier to determine from observations than $r$ (also see (Shafieloo et al. 2012; Visser 2004; Chiba \& Nakamura 2000; Arabsalmani \& Sahni 2011)). Om can also be written as a two-point diagnostic (Shafieloo et al. 2012)

$$
O m\left(z_{i} ; z_{j}\right)=\frac{\tilde{h}^{2}\left(z_{i}\right)-\tilde{h}^{2}\left(z_{j}\right)}{\left(1+z_{i}\right)^{3}-\left(1+z_{j}\right)^{3}},
$$

with $\operatorname{Om}(z ; 0)$ defined in (1). Consequently, if the Hubble parameter is known at two or more redshifts then $\operatorname{Om}\left(z_{i} ; z_{j}\right)$ can be reconstructed and one can address the issue of whether DE is the cosmological constant or not. Recent observations of BAO's in the SDSS catalogue have paved the way for reconstructing $O m$ by determining statistically independent values of $H(z)$ at several redshifts (Delubac et al. 2014). Using their determination of $H(z=2.34)=222 \pm 7 \mathrm{~km} / \mathrm{sec} / \mathrm{Mpc}$, Delubac et al. (2014) reported a surprising 2-2.5 $\sigma$ tension with the predictions of standard $\Lambda$ CDM with bestfit Planck parameters. In this paper we revisit this inconsistency using a null diagnostic approach involving an improved version of $\mathrm{Om}$. We affirm the results of Delubac et al. (2014) and also demonstrate that screened models of dark energy provide a better fit to the BAO 
data than $\Lambda$ CDM. (It may be appropriate to mention that Delubac et al. (2014) is a preprint and it is possible that these results be revised prior to publication.)

\section{DATA, METHOD \& RESULTS}

An advantage of using BAO's to deduce the nature of $\mathrm{DE}$ is that the former are measured on large scales and hence determined primarily by the linear regime of gravitational instability, a theory that has been meticulously developed and studied over the past several decades. In this letter we reconstruct $O m$ using recent determinations of $H(z)$ and attempt to answer the question as to whether DE behaves like the cosmological constant. Consider first, the following small improvement of $\mathrm{Om}$ which yields large dividents. Multiplying both sides of (2) by $h^{2}$ where $h=H_{0} / 100 \mathrm{~km} / \mathrm{sec} / \mathrm{Mpc}$, results in the improved $\mathrm{Om}$ diagnostic

$$
O m h^{2}\left(z_{i} ; z_{j}\right)=\frac{h^{2}\left(z_{i}\right)-h^{2}\left(z_{j}\right)}{\left(1+z_{i}\right)^{3}-\left(1+z_{j}\right)^{3}}
$$

where $h(z)=H(z) / 100 \mathrm{~km} / \mathrm{sec} / \mathrm{Mpc}$. A significant advantage of $O m h^{2}$ is that, for $\Lambda \mathrm{CDM}$ :

$$
O m h^{2}=\Omega_{0 m} h^{2} \text {. }
$$

Since observations of the CMB inform us that (Planck XVI 2013) $\Omega_{0 m} h^{2}=0.1426 \pm 0.0025$, it follows that for the cosmological constant $\Lambda$ :

$$
O m h^{2}=0.1426 \pm 0.0025
$$

Consequently, a departure of $O m h^{2}$ from the above value would signal that DE is not $\Lambda$. As we shall show, this is precisely what is suggested by the recent measurement of $H(z)=222 \pm 7 \mathrm{~km} / \mathrm{sec} / \mathrm{Mpc}$ at $z=2.34$ made on the basis of BAO's in the Ly $\alpha$ forest of BOSS DR11 quasars (Delubac et al. 2014).

One notes that for $n$ independent measurements of $H\left(z_{i}\right), \quad z_{i} \in z_{1} \cdots z_{n}$, the pairwise diagnostic $O m h^{2}\left(z_{i} ; z_{j}\right)$ can be determined in $\frac{n(n-1)}{2}$ different ways. In the present case $n=3$, which leads to 3 independent measurements of $O m h^{2}\left(z_{i} ; z_{j}\right)$, namely

$$
\begin{aligned}
& O m h^{2}\left(z_{1} ; z_{2}\right)=0.124 \pm 0.045 \\
& O m h^{2}\left(z_{1} ; z_{3}\right)=0.122 \pm 0.010 \\
& O m h^{2}\left(z_{2} ; z_{3}\right)=0.122 \pm 0.012
\end{aligned}
$$

where $z_{1}=0, z_{2}=0.57, z_{3}=2.34$, and the Hubble parameter at these redshifts is $H(z=0)=70.6 \pm 3.3$ $\mathrm{km} / \mathrm{sec} / \mathrm{Mpc}($ Efstathiou 2014), $H(z=0.57)=92.4 \pm$ $4.5 \mathrm{~km} / \mathrm{sec} / \mathrm{Mpc}$ (Samushia et al. 2013) and $H(z=$ $2.34)=222 \pm 7 \mathrm{~km} / \mathrm{sec} / \mathrm{Mpc}$ (Delubac et al. 2014).

One notes from (6) that the model independent value of $O m h^{2} \simeq 0.12$ is quite stable, and is in tension with the $\Lambda$ CDM-based value $\left.O m h^{2}\right|_{\Lambda \mathrm{CDM}} \simeq 0.14$. For the pair $O m h^{2}\left(z_{1} ; z_{3}\right)$ and $O m h^{2}\left(z_{2} ; z_{3}\right)$ the tension with $\Lambda$ is at over $2 \sigma$.

We should note here that these results are quite robust and not unduly sensitive to the value of $H(z=0)$. Assuming $H(z=0)=73.8 \pm 2.4 \mathrm{~km} / \mathrm{sec} / \mathrm{Mpc}$, which is the best estimated value by Riess et al. (2011), results in $O m h^{2}(0 ; 2.34)=0.121 \pm 0.009$. While using
$H(z=0)=67.1 \pm 1.2$, which is the best fit value for Hubble parameter from Planck concordance $\Lambda$ CDM model, results in $O m h^{2}(0 ; 2.34)=0.123 \pm 0.009$. Hence it is clear that the 'final' value of $H(z=0)$ should not affect the derived value of $O m h^{2}$ significantly, which suggests that our results for this quantity are robust. Likewise using the more recent SDSS galaxy BAO DR10 and DR11 result of $H(z=0.57)=96.8 \pm 3.4 \mathrm{~km} / \mathrm{sec} / \mathrm{Mpc}$ (Anderson et al. 2013) we get $O m h^{2}\left(z_{2} ; z_{3}\right)=0.120 \pm 0.010$ which is in agreement with our earlier estimations of $O m h^{2}$. This is mainly due to the high precision measurement of $H(z=2.34)$ which makes the determination of $O m h^{2}$ less sensitive to the value of $H(z)$ at lower redshifts.

Thus far our treatment has been model independent and we have refrained from commenting on the physical implications of the SDSS measurements of $H(z)$. However, as already noted in Delubac et al. (2014), these implications can be quite serious. Indeed the expansion rate at $z=2.34$, namely $H(z=2.34)=222 \pm 7 \mathrm{~km} / \mathrm{sec} / \mathrm{Mpc}$ (Delubac et al. 2014), could be in tension not only with $\Lambda$ CDM but with DE models based on the general relativistic equation $(\kappa=8 \pi G / 3)$

$$
H^{2}(z)=\kappa\left[\rho_{\mathrm{DE}}(z)+\rho_{0 m}(1+z)^{3}\right] \text { with } \rho_{\mathrm{DE}}(z) \geq 0 .
$$

Note that by setting $\rho_{\mathrm{DE}}=0$ in (7) one finds

$$
\frac{h^{2}(z)}{(1+z)^{3}}=\Omega_{0 m} h^{2} .
$$

Substituting $H(z=2.34)=222 \pm 7 \mathrm{~km} / \mathrm{sec} / \mathrm{Mpc}$ one obtains $h^{2}(z) /(1+z)^{3}=0.132 \pm 0.008$ which is somewhat lower than the CMB based value $\Omega_{0 m} h^{2}=0.142 \pm 0.002$.

This might imply one of the following: (i) $\rho_{\mathrm{DE}}(z)<0$ at high $z$ (Delubac et al. 2014; Cardenas 2014), (ii) there is non-conservation of matter so that (7) does not hold, (iii) the framework (7) is inadequate since one could be dealing with a modified gravity theory.

An example of (iii) is provided by models in which dark energy, and in particular the cosmological constant, is screened (or compensated) by a dynamically evolving counter-term. In the case of the latter, eqn. (7) is modified to

$$
H^{2}(z)=\frac{\Lambda}{3}+\kappa \rho_{0 m}(1+z)^{3}-f(z), \quad f(z)>0 .
$$

Examples of this behaviour may be found in: (i) theories in which $\Lambda$ relaxes from a large initial value via an adjustment mechanism (Dolgov 1985; Bauer et al. 2010), (ii) in cosmological models based on Gauss-Bonnet gravity (Zhou et al. 2009), and (iii) in Braneworld models (Sahni \& Shtanov 2003), etc. More generally, this behaviour occurs in modified gravity (e.g. in scalartensor gravity) when the effective gravitational constant $G_{\text {eff }}(z)<G_{\text {eff }}(0) \equiv G$, if we define $\rho_{D E}(z)$ using the present value of $\kappa$ in Eq. (7) following Boisseau et al. (2000); Sahni \& Starobinsky (2006).

A key feature of such models is that if $f(z)$ grows monotonically with redshift (but at a slower rate than $(1+z)^{3}$ in order to preserve the matter-dominated regime), then a stage will come when $\Lambda / 3$ is exactly balanced by $f(z)$, resulting in $H^{2}\left(z_{*}\right) \simeq \kappa \rho_{0 m}\left(1+z_{*}\right)^{3}$. At $z_{*}$ the effective equation of state of dark energy, $w(z)$, develops a pole, at which $\left|w\left(z_{*}\right)\right| \rightarrow \infty$. This is easily 
seen from the expression (Sahni \& Starobinsky 2006)

$$
w(x)=\frac{2 q(x)-1}{3\left(1-\Omega_{\mathrm{m}}(x)\right)} \equiv \frac{(2 x / 3) d \ln H / d x-1}{1-\left(H_{0} / H\right)^{2} \Omega_{m 0} x^{3}},
$$

where $x=1+z, \Omega_{\mathrm{m}}(x)=\Omega_{0 m} x^{3} H_{0}^{2} / H^{2}(x)$ and $q$ is the deceleration parameter. One finds from (9) and (10) that at $f\left(z_{*}\right)=\Lambda / 3$

$$
w\left(z_{*}\right)=-\frac{\left(1+z_{*}\right) f^{\prime}\left(z_{*}\right)}{H^{2}\left(z_{*}\right)-\kappa \rho_{0 m}\left(1+z_{*}\right)^{3}} .
$$

In other words, $w\left(z_{*}\right)$ diverges when $f\left(z_{*}\right)=\Lambda / 3$ and $H^{2}\left(z_{*}\right) \simeq \kappa \rho_{0 m}\left(1+z_{*}\right)^{3}$, provided $f^{\prime}\left(z_{*}\right) \neq 0$.

As a specific example of a model with this behaviour, consider the Braneworld model proposed in Sahni \& Shtanov (2003) and described, in a spatially flat universe, by the equations:

$$
\begin{aligned}
\frac{H^{2}(z)}{H_{0}^{2}}= & \Omega_{\Lambda}+\Omega_{0 m}(1+z)^{3} \\
& +2 \Omega_{l}-2 \sqrt{\Omega_{l}} \sqrt{\Omega_{0 m}(1+z)^{3}+\Omega_{\Lambda}+\Omega_{l}}, \\
\Omega_{\Lambda}= & 1-\Omega_{0 m}+2 \sqrt{\Omega_{l}},
\end{aligned}
$$

where the densities $\Omega$ are defined as :

$$
\Omega_{0 m}=\frac{\rho_{0 m}}{3 m^{2} H_{0}^{2}}, \Omega_{\Lambda}=\frac{\Lambda}{3 m^{2} H_{0}^{2}}, \Omega_{l}=\frac{1}{l_{c}^{2} H_{0}^{2}} .
$$

$l_{c}=m^{2} / M^{3}$ is a new length scale $(m$ and $M$ refer respectively to the four and five dimensional Planck masses), and $\Lambda$ is the brane tension associated with a 3 dimensional brane embedded in a $4+1$ dimensional bulk spacetime.

As shown in figure 1 the expansion rate in this model can drop below that in $\Lambda$ CDM at high $z$. It can therefore better account for the lower-than-anticipated value for $H(z=2.34)$ discussed in Delubac et al. (2014). Note also the pole in $w(z)$ at $z \simeq 2.4$. It might be mentioned that the presence of the pole in this model does not signal any pathologies since $w(z)$ is an effective equation of state. This is also true for the other theoretical models in which $w(z)$ exhibits a pole (Bauer et al. 2010; Zhou et al. 2009). Note that a pole in the equation of state may be possible to pick out in future type Ia supernova (SNIa) data sets using model independent reconstruction, as demonstrated in Shafieloo et al. (2006). Finally one might point out that although dark energy in the Braneworld behaves like a phantom it does not share the latter's pathologies (Sahni \& Shtanov 2003; Sahni 2005). The model also agrees with SNIa observations Alam \& Sahni (2006).

A detailed analysis of models with screened/compensated dark energy will be the subject of a future work.

There is another important issue that requires elaboration. The derived value of $H(z=2.34)$ given by Delubac et al. (2014) is scaled at $r_{d}=147.4 \mathrm{Mpc}$ from the Planck+WP fitting of concordance cosmology, where $r_{d}$ is the sound horizon at the drag epoch. One may argue that playing with the parameter, $r_{d}$, may help reconcile the concordance model with data. However this cannot be true since the value of $r_{d}$ used to derive $H(z=2.34)$ has been obtained assuming $\Lambda$ CDM and the discrepancy between $O m h^{2}$ and $\Omega_{0 m} h^{2}$ obtained by us is also based on $\Lambda$ CDM cosmology - see (4). One should however note that it is possible to lower the value of $r_{d}$ by increasing the expansion rate in the early Universe through the inclusion of an extra relativistic species. But this would imply a departure from the minimal standard $\Lambda \mathrm{CDM}$ model (though not in its dark energy sector).

Its also important to point out that a lower (than in $\Lambda \mathrm{CDM}$ ) value of $H(z)$ at high $z$ would affect the growth of matter density perturbations, perhaps speeding them up relative to $\Lambda$ CDM. Indeed, on scales much smaller than the horizon and within the framework of general relativity, linearized perturbations are described by the equation (Peebles 1980)

$$
\ddot{\delta}+2 H \dot{\delta}-4 \pi G \bar{\rho} \delta=0 .
$$

Clearly a lower value of $H(z)$ results in a suppression of the damping term $2 H \dot{\delta}$ (relative to $\Lambda \mathrm{CDM}$ ) and therefore to a faster growth in $\delta$. This could have important implications for structure formation which will soon be probed to great depth and accuracy by SKA, LSST, etc. However, (14) generically does not hold in modified gravity theories. In particular, in scalar-tensor gravity this equation has formally the same form at sufficiently small scales but with the effective gravitational constant $G_{\text {eff }}(t)$ instead of $G$ (Boisseau et al. 2000). Therefore a detailed analysis of perturbation growth in such models needs to be carried out before firm predictions can be made about $\delta(z)$.

\section{SUMMARY}

To summarise, this short letter demonstrates that the recent estimation of $H(z=2.34)$ from BAO observations in the SDSS DR11 data is in tension with CMB observations assuming standard $\Lambda \mathrm{CDM}$. This tension is independent of the current value of the Hubble parameter $H(z=0)$. In our analysis we have implemented an improved version of the $O m$ diagnostic, called $O m h^{2}$, which can be derived by having independent measurements of $H(z)$ at two redshifts. $O m h^{2}$ should be equal to $\Omega_{0 m} h^{2}$ if the universe corresponds to spatially flat $\Lambda$ CDM. Our estimated value of $O m h^{2} \approx 0.122 \pm 0.01$ (which should also be the value of $\Omega_{0 m} h^{2}$ for $\Lambda \mathrm{CDM}$ ) is robust against variations of the Hubble parameter $H_{0}$ and is in strong tension with $\Omega_{0 m} h^{2}=0.1426 \pm 0.0025$ given by Planck + WP.

In the absence of systematics in the CMB \& SDSS data sets, our results suggest a strong tension between concordance cosmology and observational data. Since resolving this discrepancy by changing initial conditions and/or the form of the primordial spectrum might be difficult (note that $\Omega_{0 m} h^{2}$ does not change much if one deviates smoothly from the power-law form of the primordial spectrum (Hazra et al. 2013; Hazra \& Shafieloo 2014; Hazra et al. 2014)), allowing dark energy to evolve seems to be the most plausible approach to this problem. Evolving dark energy models which might accommodate the SDSS data better than $\Lambda$ CDM include those in which the cosmological constant was screened in the past. The effective equation of state in such models develops a pole at high $z$, which emerges as a smoking gun test for such scenarios.

A.S. wishes to acknowledge support from the Korea Ministry of Education, Science and Technology, 

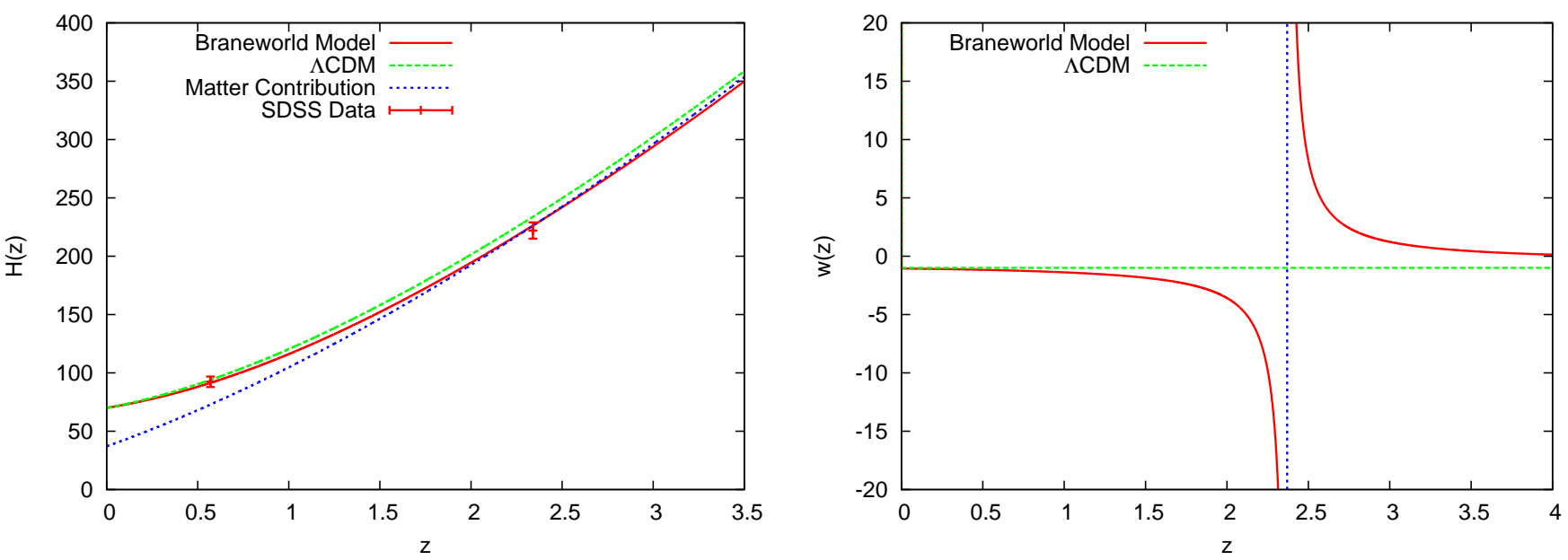

Fig. 1. - The Hubble parameter (left panel) and the effective equation of state of dark energy (right panel) are shown for the Braneworld model described by (12) (solid red) and $\Lambda$ CDM (dotted green). Also shown is the matter contribution: $H_{0} \sqrt{\Omega_{0 m}(1+z)^{3}}$ where $H_{0}=70$ $\mathrm{km} / \mathrm{sec} / \mathrm{Mpc}$ and $\Omega_{0 m}=0.28$ (dotted blue). In the Braneworld model the cosmological constant is screened in the past as a result of which the expansion rate drops below that in $\Lambda \mathrm{CDM}$ at high $z$. This feature permits the Braneworld to better account for the low value of $H(z=2.34)$ discovered in Delubac et al. (2014). Note that $H_{\text {Brane }} \simeq H_{0} \sqrt{\Omega_{0 m}(1+z)^{3}}$ at $z \simeq 2.4$. The associated pole in $w(z)$ at $z \simeq 2.4$ is shown in the right panel. The parameters for the Braneworld model are $\Omega_{0 m}=0.28$ and $\Omega_{\ell}=0.025$ in (12).

Gyeongsangbuk-Do and Pohang City for Independent Junior Research Groups at the Asia Pacific Center for Theoretical Physics. A.S. would like to acknowledge the support of the National Research Foundation of Korea
(NRF-2013R1A1A2013795). A.A.S. was partially supported by the grant RFBR 14-02-00894 and by the Scientific Programme "Astronomy" of the Russian Academy of Sciences.

\section{REFERENCES}

Ade, P. et al., 2013, Planck 2013 results. XVI. Cosmological parameters, arXiv: 1303.5076

Alam, U., Sahni, V., Saini, T. D. \& Starobinsky, A. A. 2003, Mon. Not. Roy. Ast. Soc. 344, 1057

Alam, U. \& Sahni, V. 2006, Phys.Rev. D73, 084024

Anderson, L. et al., 2014, MNRAS 441, 24

Arabsalmani, M. \& Sahni, V. 2011, Phys. Rev. D83, 043501

Bauer, F., Sola, J. \& Stefancic, H. 2010, JCAP 1012, 029

Boisseau, B., Esposito-Farese, G., Polarski, D. \& Starobinsky, A. A. 2000, Phys. Rev. Lett. 85, 2236

Cardenas, V. H. 2014, arXiv:1405.5116

Carroll, S. M. 2001, Living Rev.Rel. 4, 1

Chiba, T. \& Nakamura, T. 1998, Prog. Theor. Phys. 100, 1077

Chiba, T. \& Nakamura, T. 2000, Phys. Rev. D62, 121301(R)

Clifton, T., Ferreira, P. G., Padilla, A. \& Skordis, C. 2012, Phys.

Rep. 513, 1.

Copeland, E. J., Sami, M. \& Tsujikawa, S. 2006, Int. J. Mod. Phys. D 15, 1753

Delubac, T. et al., 2014, arXiv:1404.1801

Dolgov, A. D. 1985, JETP Lett. 41, 345

Efstathiou, G. 2014, MNRAS 440, 1138

Hazra, D. K., Shafieloo, A. \& Smoot, G. F. 2013, JCAP 1312, 035

Hazra, D. K. \& Shafieloo, A. 2014, JCAP 1401, 043

Hazra, D. K., Shafieloo, A., Smoot, G. F. \& Starobinsky, A. A. 2014, JCAP 1406, 061

Padmanabhan, T. 2003, Phys. Rep. 380, 235
Peebles, P.J.E. 1980, The Large scale structure of the universe, Princeton University press

Peebles, P. J. E. \& Ratra, B. 2003, Rev. Mod. Phys. 75, 559

Riess, A. G., Macri, L. \& Csertano, S. 2011 ApJ730, 119

Sahni, V. \& Starobinsky, A. A. 2000, Int. J. Mod. Phys. D 9, 373

Sahni, V., Saini, T. D., Starobinsky, A. A. \& Alam, U. 2003, JETP Lett. 77, 201

Sahni, V. \& Shtanov, Yu. 2003, JCAP 0311, 014

Sahni, V. 2004, Lect. Notes Phys. 653, 141

Sahni, V. 2005, astro-ph/0502032.

Sahni, V. \& Starobinsky, A. A. 2006, Int. J. Mod. Phys. D 15, 2105

Sahni, V., Shafieloo, A. \& Starobinsky, A. A. 2008,

Phys. Rev. D78, 103502

Samushia, L. et al., 2013, MNRAS 429, 1514

Shafieloo, A., Alam, U., Sahni, V. \& Starobinsky, A. A. 2006, MNRAS 366, 1081

Shafieloo, A., Sahni, V. \& Starobinsky, A. A. 2012,

Phys. Rev. D86, 103527

Shafieloo, A. 2014, Nucl.Phys.Proc.Suppl. 246-247, 171

Visser, M. 2004, Class. Quant. Grav. 21, 2603

Zhou, S-Y., Copeland, E. J. \& Saffin, P. M. 2009, JCAP 0907, 009

Zunckel, C. \& Clarkson, C. 2008, Phys. Rev. Lett 101, 181301 